\title{
Methotrexate-Associated Follicular Lymphoma
}

National Cancer Institute

\section{Source}

National Cancer Institute. Methotrexate-Associated Follicular Lymphoma. NCI

Thesaurus. Code C7254.

A follicular lymphoma that develops in patients who are immunosuppressed with methotrexate. 